\title{
EFECTIVIDAD Y EFICIENCIA DE LOS CANALES DE BÚSQUEDA DE EMPLEO EN COLOMBIA*
}

\author{
Lina María Restrepo Plaza** \\ Recibido: abril 25 de 2012 • Aceptado: mayo 24 de 2013
}

\section{RESUMEN}

El mercado laboral no puede definirse como una institución en la cual confluyen oferentes y demandantes para negociar una mercancía a un precio determinado; se ha identificado que existen distintos mecanismos, que son canales de búsqueda, mediante los cuales los agentes logran emparejar su capacidad de trabajar con las vacantes disponibles. Para verificar la efectividad y la eficiencia de estos canales de búsqueda se utilizó la información de la Gran Encuesta Integrada de Hogares, para las 13 áreas metropolitanas de Colombia del segundo trimestre de 2008; se realizaron estimaciones de modelos paramétricos de duración y elección discreta y otros modelos no paramétricos tipo Kaplan-Meier que permitieron verificar que las búsquedas informales, es decir, aquellas realizadas a través de familiares y amigos, son más efectivas y eficientes que los procesos de selección formales.

\section{PALABRAS CLAVE}

Empleo, canales de búsqueda de empleo, duración del desempleo.

\section{CLASIFICACIÓN JEL}

E24, J64

\section{CONTENIDO}

Introducción; 1. Estado del arte; 2. Marco teórico; 3. Resultados exploratorios; 4. La efectividad de los canales de búsqueda de empleo; 5 . La eficiencia de los canales de búsqueda: los modelos de duración; 6 . Las tareas pendientes del mercado laboral colombiano; 7. Conclusiones; Bibliografía; Anexos.

\footnotetext{
Este artículo de investigación es el resultado de la tesis de maestría de la autora como Magíster en Economía Aplicada de la Universidad del Valle. La tesis fue asesorada por el profesor José Ignacio Uribe durante agosto del 2010 y junio de 2011. La autora agradece a José Ignacio Uribe, Boris Salazar, Jaime Tenjo y a Jorge Mario Uribe, por sus aportes al mejoramiento del documento. También agradece a Paula Rivas por su apoyo en el trabajo editorial para la presentación a la revista.

** Economista, Universidad del Valle, Cali, Colombia. Ms en Economía Aplicada, Universidad del Valle, Cali, Colombia. Profesora del Departamento de Economía de la Universidad del Valle, Cali, Colombia. Miembro de los grupos de investigación: Conflicto, Aprendizaje y Teoría de Juegos y Desarrollo Económico, Crecimiento y Mercado Laboral. Correo electrónico: lina.restrepo@correounivalle.edu.co
} 


\section{EFFECTIVENESS AND EFFICIENCY OF JOB SEARCH CHANNELS IN COLOMBIA}

\section{ABSTRACT}

The labor market cannot be defined as an institution where buyers and sellers come together to negotiate a commodity at a given price; therefore three different search channels mechanisms have been identified where the agents are able to match the working abilities with the available jobs. In order to verify the effectiveness and efficiency of these search channels, the Household Integrated Survey information was used for the 13 metropolitan areas of Colombia in the second quarter of 2008. Parametric estimations where made such as the duration and discreet choice models and other non parametric model estimations such as Kaplan-Meier which proved that informal searches, meaning by these those done to family members and friends, are more effective and efficient than the formal selection processes.

\section{KEY WORDS}

Employment, job search channels, duration of unemployment

\section{JEL CLASSIFICATION}

E24, J64

\section{CONTENT}

Introduction; 1. State of art; 2 . Theoretical framework; 3 . Exploratory results; 4 . The efficacy of job search channels; 5 . The efficiency of job search channels; Duration models; Pending tasks of the Colombian labor market; 7. Conclusions; Bibliography; Attachments.

\section{EFECTIVIDADE E EFICIÊNCIA DOS CANAIS PARA PROCURA DE EMPREGO NA COLÔMBIA}

\section{RESUMO}

O mercado laboral não pode definir-se como uma instituição na qual confluem oferentes e demandantes para negociar uma mercadoria a um preço determinado; tem se identificado que existem distintos mecanismos, que são canais de busca, por meio dos quais os agentes conseguem equiparar sua capacidade de trabalho com as vacantes disponíveis. Para verificar a efetividade e a eficiência destes canais de busca utilizou-se a informação da Grande Enquete Integrada de Lares, para as 13 áreas metropolitanas da Colômbia do segundo trimestre de 2008; realizaram-se estimações de modelos paramétricos de duração e eleição discreta e outros modelos não paramétricos tipo Kaplan-Meier que permitiram verificar que as busca informais, é dizer, aquelas realizadas por meio de familiares amigos, são mais efetivas e eficientes que os processos de seleção formais.

\section{PALAVRAS CHAVE}

Emprego, canais de procura de emprego, duração do desemprego.

\section{CLASIFICAÇÃO JEL}

E24, J64

\section{CONTEÚDO}

Introdução; 1. Estado da arte; 2. Marco teórico; 3. Resultados exploratórios; 4. A eficácia dos canais de procura de emprego; 5 . A eficiência dos canais de busca: os modelos de duração; 6 . As tarefas pendentes do mercado laboral colombiano; 7. Conclusões; Bibliografia; Anexos 
Efectividad y eficiencia de los canales de búsqueda de empleo en Colombia

\section{INTRODUCCIÓN}

A finales del año 2009 la tasa de desempleo nacional era del 12\%, un punto porcentual por encima del cierre de 2008; quien hizo parte de esta estadística, estuvo expuesto a preguntas incómodas como: "¿ha buscado empleo?", "¿ha enviado hojas de vida?", "¿conversó con fulanito de tal para que le ayude?", "¿compró el periódico del domingo para ver los clasificados?"; lo que no sabe el curioso es que quien busca empleo seguramente ha agotado estas y más posibilidades, pero no ha conseguido que lo contraten.

Los canales de búsqueda son el mecanismo mediante el cual las personas buscan y encuentran empleo, algunos en mayor y otros en menor tiempo. La efectividad de un canal de búsqueda es la posibilidad que este ofrece para que un individuo encuentre empleo; la eficiencia es la capacidad que tiene de hacerlo en el menor tiempo posible.

Viáfara y Uribe (2009, p. 15) identificaron que en el año 2006, el 87,8\% de los desempleados y el 60,4\% de los empleados en Colombia utilizan canales de búsqueda informales para encontrar empleo como lo son amigos y familiares; el 9.3\% y el $26,6 \%$, respectivamente, lo hacen en canales informales moderados como las visitas personales a empresas, y el 2,9\% y 13\% utilizan canales formales como las convocatorias, los clasificados entre otros. Viáfara y Uribe (2009, p. 17) afirman:

De quienes utilizan canales formales e informal moderado 3 de cada 4, es decir un $75 \%$, han salido del desempleo a los 12 meses o menos. En contraste, de los que utilizan canales informales 3 de cada 4 necesitan 5 años para salir del desempleo. Esta es otra aproximación a la mayor efectividad de los canales formales e informal moderado. Es decir, los individuos que utilizan canales más modernos y meritocráticos logran empleo de manera más rápida en contraste con los que utilizan redes sociales únicamente.

En ese orden de ideas, pareciera que no siempre los canales de búsqueda más utilizados son los más eficientes, por lo que podría pensarse que los canales de búsqueda de empleo, al igual que los canales electromagnéticos, tienden a saturarse, lo que genera una posible competencia entre los individuos que se encuentran realizando la búsqueda en cada uno de ellos. ¿Quiénes ganan esta competencia? es una pregunta que se contesta en este trabajo.

Es posible que, si los buscadores conocieran esta información hicieran una búsqueda más intensiva en los canales más eficientes y/o más eficaces; no obstante, debe ser consciente de que la efectividad y la eficiencia no son del todo exógenas, sino que también dependen de las características sociales, económicas y demográficas del individuo. 
Este estudio identifica la incidencia del entorno socioeconómico y de otras variables demográficas de los individuos en la eficiencia y la efectividad de los canales de búsqueda que estos utilizaron para encontrar empleo en las 13 áreas metropolitanas en el segundo trimestre de 2008 con información de la Gran Encuesta Integrada de Hogares (GEIH) de este año. Para realizar inferencia acerca de la eficiencia de los canales de búsqueda, entendida esta como la posibilidad de ocuparse en determinado período de tiempo, se realizaron estimaciones paramétricas y no paramétricas de la duración del desempleo, utilizando los canales de búsqueda como parámetro -en el modelo paramétrico de duración ${ }^{1}$ - o como variable de identificación -en el modelo no paramétrico tipo Kaplan-Meier ${ }^{2}$. Para dar cuenta de la efectividad, es decir, de la posibilidad de conseguir empleo, independientemente de la duración de la búsqueda, se estimó un modelo de elección discreta tipo logit³, en el cual, tanto los canales de búsqueda como la información del individuo se consideraron variables de control.

A partir de las estimaciones realizadas con información de la Gran Encuesta Integrada de Hogares (GEIH) del segundo trimestre de 20084, se encontró que los canales de búsqueda informales suelen ser más eficaces y más eficientes que los formales, efecto que aumenta con años de educación, la edad y el hecho de ser jefe de hogar.

El documento inicia con esta introducción, seguida por un estado del arte en el cual se exponen estudios que, de alguna manera, también han intentado responder al objetivo que este trabajo persigue. Luego se plantea el marco teórico que fundamenta el proceso de búsqueda de empleo, se desarrolla una descripción de los buscadores y se hacen estimaciones que permiten concluir sobre la efectividad y la eficiencia de los canales de búsqueda. Para finalizar, se identifican algunos vacíos en el mercado laboral colombiano y se proponen lineamientos de política que podrían reducir la inoperancia del mismo.

Kiefer (1988, p. 650) propone la estimación de modelos de duración paramétricos a través de una función de riesgo la cual resulta una alternativa legítima para la especificación de la función de densidad en presencia de datos censurados.

2 Kaplan y Meier (1958, p. 458) proponen un tipo de modelos que calculan la proporción de individuos que sobreviven a determinado evento, en este caso desempleo, en un período de tiempo.

3 Los modelos logit permiten inferir sobre el efecto que tiene un conjunto de variables sobre la probabilidad de ocurrencia de un evento, para este artículo el evento se refiere a la probabilidad de ocuparse. Para conocer sobre las especificaciones de este tipo de modelos, el lector puede remitirse a Greene (2003, p. 663)

4 El lector puede tener acceso a los microdatos utilizados en este estudio en la página oficial del Departamento administrativo Nacional de Estadística, DANE. 
Efectividad y eficiencia de los canales de búsqueda de empleo en Colombia

\section{ESTADO DEL ARTE}

\section{1. ¿Qué se ha dicho sobre la eficiencia de la búsqueda?: la duración del desempleo}

En un principio, los estudios sobre duración del desempleo fueron abordados a partir de un análisis que solo incluía a los sujetos que se encontraban en búsqueda de empleo bajo el supuesto de que estos encontraban empleo el mismo día que contestaban la encuesta; de ahí que se presentaban posibles subestimaciones en los parámetros calculados. A pesar de esta limitación, fueron el inicio de una amplia literatura interesada en estudiar este fenómeno; de ahí que vale la pena mencionar el trabajo de Tenjo y Ribero (1998, p. 28) quienes desarrollan un análisis de los determinantes de la duración que incorpora variables del individuo, de su entorno familiar ${ }^{5}$ y el tipo de contrato firmado en su último período laboral. De acuerdo con los resultados, la duración del desempleo es mayor para las mujeres que para los hombres; los jóvenes presentan una mayor tasa de entrada al desempleo pero la duración del mismo es corta, mientras que los de edad más avanzada tienen pocos períodos de desempleo, pero de larga duración. Por último, los autores encontraron que los cambios en la duración del desempleo, que ya se visualizaban a mediados de 1990, parecían estar asociados más a cambios en los niveles de la actividad económica que a cambios en las características de los desempleados, por lo cual podría asumirse que en la década de 1990 la duración del desempleo estaba determinada por factores de la demanda laboral que eran consecuencia directa del desempeño del ciclo económico.

Por otra parte, Castellar y Uribe (2003, p. 16) superan el sesgo mencionado y realizan un análisis sobre la duración del desempleo con datos no censurados, es decir, consideran la información revelada por los empleados. Los autores estiman modelos de duración paramétricos (función Weibull) con base en la Encuesta Nacional de Hogares (ENH) del II trimestre de los años 1988, 1992, 1994, 1996 y 1998, la cual compararon con información suministrada por la base de datos del $\mathrm{CIE}^{6} \mathrm{del}$ SENA. Los hallazgos sugirieron que la duración del desempleo, que presentaba una reducción entre 1988 y 1994, empezó a incrementarse desde 1996 hasta alcanzar un promedio de 42 semanas para 1998. Dentro de los determinantes que afectan de manera inversa la duración del desempleo se identificaron: la jefatura de hogar, bajos niveles de ingresos no laborales, ser hombre, mayores niveles de educación (aunque los efectos no son lineales), la experiencia y una menor dispersión salarial (a

\footnotetext{
5 El ingreso del resto de la familia, la educación, la edad, estado civil, la experiencia laboral previa y los ingresos no laborales.

6 Centro de Información para el Empleo.
} 
Lina María Restrepo Plaza

un individuo con el mismo nivel de calificación le pueden ofrecer distintos salarios, razón por la cual la búsqueda es rentable) ${ }^{7}$.

\section{2. ¿Qué se ha dicho sobre la efectividad y la eficiencia de los canales de búsqueda de empleo?}

Reid (1972, p. 479) considera las decisiones de búsqueda de empleo a partir de los canales utilizados para tal fin; es por ello que propone una caracterización de los canales de búsqueda de la siguiente manera: formales, que incluyen las agencias de empleo y las convocatorias, y los informales, que consideran el acceso a información privilegiada a través de amigos y familiares. El autor elabora un estudio de la eficiencia de los distintos canales haciendo uso de la información del medio oeste de Inglaterra entre 1966 y 1968. Se encuentra que los métodos informales pueden ser más eficientes que los formales y, en particular, reportan una mayor eficiencia en los casos de reubicación laboral voluntaria, en donde ofrecen oportunidades de mejores salarios y empleos acordes con las preferencias del trabajador.

Roshchin y Markova (2004, p. 12) llevaron a cabo un estudio cuyos objetivos principales eran identificar los determinantes de la elección de las estrategias de búsqueda de empleo y la efectividad de los mismos en el mercado laboral ruso, teniendo en consideración las decisiones de empleados y desempleados captadas en 6 rondas de la encuesta longitudinal para el monitoreo de Rusia en los años 19941996, 1998, 2000, y 2001. De manera novedosa, los autores proponen la construcción de 5 estrategias para la búsqueda de empleo que consisten en la combinación pura o mixta entre canales formales e informales. En esta investigación, la efectividad de la búsqueda depende de la estrategia elegida. A partir de un análisis factorial tipo componentes principales, se identificó que suelen ser más efectivas las estrategias que incluyen la utilización de redes informales o un mayor número de canales de búsqueda. Se identificó, además, que el principal determinante de la elección de los individuos por una estrategia es el retorno esperado por el empleo, más que las restricciones presupuestales de la búsqueda.

En algunos países desarrollados el Estado genera incentivos que inciden de manera directa sobre la duración del desempleo; de ahí que sea necesario monitorear a los individuos que reciben un apoyo estatal y evitar así ineficiencias en la asignación de los recursos públicos. Van den Berg y Van der Klaauw (2007, p. 5) realizaron un experimento para cuantificar el efecto que tiene el programa de Consejería y

Castellar y Uribe (2003, pág. 18) definen dispersión salarial "como el coeficiente de variación salarial (CVSALi) del sector donde el individuo está buscando. Teóricamente se puede anticipar que en un sector de mayor dispersión habrá mayor probabilidad de recibir ofertas más altas lo cual, con agentes neutrales al riesgo, aumentará el tiempo de búsqueda" 
Efectividad y eficiencia de los canales de búsqueda de empleo en Colombia

Monitoreo de los Países Bajos el cual tiene por objetivo reducir la duración del desempleo y, por lo tanto, disminuir los desembolsos de seguros al desempleo. Los autores utilizaron un modelo de búsqueda caracterizado por emplear múltiples canales y esfuerzos endógenos de búsqueda. El modelo estructural estimado contó con una muestra de 393 individuos desempleados que recibieron seguros de desempleo entre el 24 de agosto y el 2 de diciembre de 1998. Los resultados fueron coherentes con la intuición económica: el monitoreo reduce la duración del desempleo, pero genera un efecto negativo sobre la calidad del empleo que aceptan los trabajadores; esto se observa a través de los salarios percibidos por los individuos que se encuentran monitoreados por el programa; por esta razón, los autores proponen un cambio hacia una política de bonos de reubicación laboral, en lugar de los seguros del desempleo.

Dentro de las características del individuo que pueden incidir en que este permanezca en una situación de desempleo se encuentran los canales de búsqueda utilizados para engancharse. Uribe, Viáfara y Oviedo (2007, p. 46) definen tres tipos de canales de búsqueda y analizan la efectividad de cada uno en el mercado laboral colombiano haciendo uso de la Encuesta de Calidad de Vida de 2003. Los canales definidos por los autores son: canales formales, canales informales y canales informales moderados. Los primeros son de dominio público y de libre acceso; los segundos son derivados del capital social de los individuos que tienen contactos con capacidad de decisión en las empresas que buscan un enganche; los últimos también responden a las redes sociales de cada sujeto, pero sin que el contacto implique una decisión de contratación. El análisis consiste en definir una tasa de efectividad de cada canal como instrumento para calcular su eficiencia; esta tasa se mide como la razón entre el porcentaje de ocupados y desocupados que buscan por el mismo canal. Los resultados muestran que si bien los canales informales son los más utilizados en el mercado laboral (78,8\% de los ocupados y 55,2\% de los desocupados), su efectividad se encontró por debajo de la efectividad de los canales formales. La efectividad de los canales informales e informales moderados se incrementa a medida que aumentan la edad y el nivel de educación debido a la relación que tienen estas características con las posibilidades de establecer redes sociales con las instancias de decisión de contratación laboral de las empresas.

Oviedo (2007, p. 15) analiza el problema del buscador, la duración del desempleo que experimenta y el impacto de la utilización de los diferentes canales de búsqueda, en el tiempo que el individuo permanece desempleado, utilizando la Encuesta de Calidad de Vida de 2003. La autora realiza una estimación de modelos de elección múltiple que le permiten verificar la propensión de los agentes a utilizar un canal de búsqueda en particular; su análisis es complementado con una estimación de un modelo de supervivencia para captar el efecto de los canales de búsqueda en 
la duración del desempleo. Oviedo (2007, p. 1) "encuentra que los canales que reducen el tiempo de duración del desempleo son aquellos que implican la participación activa del buscador" es decir, los canales informales moderados. En cuanto a la elección de canales de búsqueda y a la probabilidad de emplearse por los mismos se encontró que, a medida que aumenta el nivel de educación aumenta la probabilidad de elegir y encontrar trabajo mediante métodos formales e informales moderados. Por último, de acuerdo con los resultados de la autora, puede afirmarse que pertenecer a los estratos medio (3 y 4) y alto (5 y 6) incrementa la probabilidad de buscar y encontrar empleo a partir de métodos informales, e informales moderados.

Tasci (2008, p. 13) realizó una investigación con objetivos similares a los del estudio ruso. El objetivo principal de la investigación era examinar los factores que influyen sobre la intensidad de la búsqueda de empleo en Turquía, para lo cual utilizaron la información arrojada por la Encuesta de Fuerza Laboral de los Hogares para los años 2000 y 2001. Se realizó una estimación tipo Heckman en dos etapas -la cual es afectada por la censura observada por solo trabajar con individuos desempleados- que sugirió que las mujeres y las personas que viven en áreas menos urbanizadas presentan una mayor intensidad en la búsqueda de empleo debido a variables socio-demográficas como la educación, la cualificación y la experiencia laboral. Una vez más se reconoció que los principales canales de búsqueda utilizados responden a métodos informales, y que mecanismos formales y públicos suelen ser de baja utilización.

En relación con la duración del desempleo, Du, Yang y Dong (2007, p. 21) utilizaron la Encuesta Nacional de Hogares de China de 2003 con el objetivo de realizar un análisis sistemático de las razones por las cuales las mujeres chinas permanecen más tiempo en situación de desempleo; para ello realizaron una estimación por máxima verosimilitud de una función de duración tipo Weibull a partir de variables asociadas al capital humano, a las condiciones de ingresos del hogar y los canales de búsqueda empleados. El estudio reveló que gran parte de los esfuerzos de búsqueda de las mujeres se ve obstaculizado por 1) la carencia de redes sociales, 2) prejuicios contra las mujeres casadas, 3) acceso desigual a los servicios de reempleo, y 4) discriminación salarial en el mercado laboral.

Viáfara y Uribe (2009, p. 150) desarrollaron un estudio sobre la duración del desempleo en Colombia con información de la Encuesta Continua de hogares (ECH) del segundo trimestre de 2006. Los autores consideran la duración del desempleo como un indicador de efectividad de los canales de búsqueda de empleo lo cual no había sido considerado antes en los modelos de duración. El modelo propuesto captura, por una parte, el efecto aspiraciones a través de variables asociadas al salario de reserva que son aquellas incluidas en una ecuación minceriana ampliada con la 
Efectividad y eficiencia de los canales de búsqueda de empleo en Colombia

jefatura de hogar, y por otra parte, el efecto oportunidades, que se aborda a través de los canales de búsqueda de empleo. Los autores estimaron modelos paramétricos (Weibull de riesgos proporcionales) y no paramétricos (Kaplan- Meier) de duración. Los modelos no paramétricos arrojaron, mediante una función de supervivencia en situación de desempleo, que aproximadamente el 54\% de los sujetos encuestados se han convertido en desempleados de larga duración (mayor o igual a un año), situación que se agudiza por la utilización de canales informales. Por otra parte, en relación con la efectividad de los canales de búsqueda, los 4 modelos paramétricos estimados arrojaron que si bien los datos descriptivos mostraron más utilización de los canales informales, la duración del desempleo es mayor para quienes utilizan las redes sociales como mecanismos de búsqueda de empleo, lo cual implica una menor efectividad de este tipo de canales.

\section{MARCO TEÓRICO}

\subsection{Factores que explican el desempleo}

Existe un amplio conjunto de teorías que intentan explicar el desempleo; gran parte de ellas hace alusión a las rigideces del mercado laboral como una explicación a la baja demanda laboral. Son ejemplos de estas teorías los salarios de eficiencia desarrollados por Weiss (1980, p. 530) en los que se afirma que un salario más alto permite resolver los inconvenientes derivados de la información asimétrica, ya que este atraerá a los trabajadores más productivos y podrá realizar una mejor selección; en este modelo la firma no busca encontrar el mínimo valor del salario para suplir su demanda condicionada de trabajadores, sino encontrar el valor mínimo para contratar trabajo eficiente. Los salarios de eficiencia se consideran una rigidez del mercado laboral ya que, dado que el salario es entendido como una medida de productividad, las firmas no están dispuestas a reducir el salario que pagan y así poder contratar más trabajadores; esto genera desempleo en la población menos educada y con menor experiencia de la sociedad.

Lindbeck y Snower (1986, p. 235 y 1988, p. 365) desarrollan la teoría de los insider-outsider la cual ofrece una explicación intuitiva acerca del funcionamiento de los sindicatos en el mercado laboral. Los sindicatos están conformados por insiders que son individuos empleados con posibilidad de incidir en el proceso de determinación de los salarios, mientras que los outsiders son desocupados que carecen de esa posibilidad. El salario determinado deja por fuera a un grupo de la población que estaría dispuesto a emplearse por una remuneración menor; no obstante, las normas sociales en las que se circunscriben ${ }^{8}$ les impiden llevar a cabo una puja para

8 Estas normas sociales hacen referencia a la posibilidad de ser aceptado en su entorno laboral y de 
ser contratados. El desempleo que se deriva de esta teoría afecta, en particular, a la población menos educada y con menos experiencia, es decir, aquella que se encuentra en el lado opuesto de la explicada por los salarios de eficiencia.

Pissarides (2009, p. 1340) reconoce que existen rigideces que obedecen a la negociación del salario que impiden renegociarlo en los trabajos en curso, pero demuestra, de manera empírica, que no existe evidencia acerca de su efecto sobre el proceso de búsqueda y la ubicación laboral. Es por ello que converge a lo expuesto por Mortensen y Pissarides (1994, p. 413) acerca de que el proceso de creación de empleos es una decisión de la firma, la cual no se encuentra relacionada de forma directa con la negociación del salario de los insiders; por lo tanto, las rigideces que sí pueden influir en la generación de empleo son las generadas por la falta de emparejamiento entre las demandas de las empresas y la oferta laboral.

\subsection{Cómo se explica el proceso de búsqueda de empleo}

Hasta ahora, las teorías de la búsqueda de empleo han abordado la elección de una estrategia óptima de búsqueda que puede redundar según Stigler (1961, p. 213), en la identificación de un númerode empresas a consultar, o según Mortensen y Pissarides (1998, p. 6) en una regla de decisión para finiquitar la búsqueda.

El modelo de Stigler (1961, p. 213) presenta asimetrías de información asociadas a las tasas salariales que están dispuestos a pagar diferentes patronos, razón por la cual, visto desde una perspectiva microeconómica, un sujeto debe realizar una búsqueda óptima que le garantice tener un conjunto de información para reducir la incertidumbre y poder elegir un salario satisfactorio. El autor desarrolló un modelo de búsqueda óptima que considera los costos positivos que esta tiene, lo cual incentiva a acotar el tamaño de la muestra. Martín (1995, p. 43) reconoce que, si bien el modelo es estilizado desde su fundamentación microeconómica puede presentar resultados por debajo del óptimo, ya que para un tamaño óptimo de $n^{*}$ empresas muestreadas, es posible que la mejor oferta salarial haya sido identificada en $n^{*}-k$, y debido a las mismas asimetrías de información que dieron origen a este modelo, el trabajador deberá continuar la búsqueda hasta completar su tamaño de muestra óptimo.

Con el objetivo de superar la posible ineficiencia derivada de una muestra de empresas fija, Mortensen y Pissarides $(1998$, p. 6) proponen un modelo de búsqueda secuencial en el cual no se determina el tamaño óptimo del número de empresas a muestrear, sino la estrategia óptima para terminar la búsqueda; esta se concluye una

recibir cooperación por parte de los demás trabajadores. Si esto no ocurre tanto los trabajadores como los empresarios estarían perdiendo; los primeros perderían en términos de remuneración y calidad del empleo, y los segundos perderían productividad. 
Efectividad y eficiencia de los canales de búsqueda de empleo en Colombia

vez se acepta un empleo que cubra su salario de reserva y los costos acumulados de la búsqueda hasta el momento. En ese sentido, los autores proponen una regla de decisión que bien podría compararse con la elección interactiva propia de la Teoría de Juegos si se consideraran los costos de las empresas en un proceso de selección.

En ese orden de ideas, se presenta una expresión genérica del principio básico de la programación dinámica llamado el principio de optimalidad de Bellman el cual es citado por Oviedo (2002, p. 5) argumentando que "una política óptima tiene la propiedad de que, cualesquiera sean el estado y las decisiones iniciales tomadas (es decir, el control), las restantes decisiones deben constituir una política óptima con independencia del estado resultante de la primera decisión".

Esto quiere decir que una vez el individuo toma la decisión de interrumpir la búsqueda, esta decisión será óptima para cualquier instante $t+1$.

$\mathrm{U}_{t=\frac{6-a}{1+r}+\frac{1}{1+r} \int \max \left\{w, \mathrm{U}_{t+1}\right\} d \mathrm{~F}(\mathrm{~W})} \quad t=1,2, \ldots$

De acuerdo con Mortensen y Pissarides (1998, p. 12), $U_{t}$ representa el valor de la búsqueda de empleo, $b$ es el flujo de ingreso percibido por los agentes mientras se encuentran desempleados, $a$ es el costo de búsqueda de empleo por período y W representa el valor generado por una oferta contractual. La estrategia óptima requiere encontrar aquel valor del contrato, $\mathrm{W}_{\mathrm{t}}$, que supera el valor de la búsqueda en un período posterior, $\mathrm{U}_{\mathrm{t}+1}$; por tal razón la búsqueda se detiene en el punto en el cual $\mathrm{W}_{\mathrm{t}}>\mathrm{U}_{\mathrm{t}+1}$

Al igual que los modelos antes planteados, el modelo que aquí se abordó intenta reducir los niveles de incertidumbre con los cuales los buscadores se enfrentan al mercado laboral, con la particularidad de que la información relevante para encontrar empleo intenta recopilarse mediante la selección de un canal de búsqueda óptimo; de acuerdo con las características del individuo, este canal le permitirá no solo obtener empleo sino, además, hacerlo en el menor tiempo posible.

\section{RESULTADOS EXPLORATORIOS}

Para efectos de los cálculos exploratorios y las estimaciones se empleó la siguiente agrupación de los canales de búsqueda:

- Canales formales: en la GEIH se pudieron identificar canales como llevar la hoja de vida a las empresas, agencias de contratación temporal, o servicios públicos de empleo, como los Centros de Información para el Empleo del SENA, o de otra clase: avisos clasificados, convocatorias, Internet, etc. 
- Canales informales: en la GEIH se identifican a través de las categorías de ayuda o información de familiares, amigos y colegas, y realizar gestiones para establecer su propio negocio.

Se realizó una descripción de los buscadores de empleo que incluye tanto a desempleados como a empleados a partir de variables socioeconómicas y demográficas como el sexo, la jefatura de hogar, el nivel educativo y el estrato socioeconómico. Al cruzar estas variables con la utilización de los canales de búsqueda y con la duración media del desempleo, se logró construir un conjunto de intuiciones básicas de la relación que puede existir entre estas variables en la efectividad y la eficiencia de los canales de búsqueda; intuiciones que luego fueron complementadas con las estimaciones econométricas.

En términos demográficos, la población económicamente activa (PEA) se caracteriza por ser un 53\% femenina y un 47\% masculina. El 35\% de las personas son jefes de hogar y de ellos, el 66\% son hombres y el 34\% son mujeres. El 37\% de la PEA presenta un nivel educativo que no supera la Básica Secundaria, mientras que el 63\% ha alcanzado niveles de Educación Media y Superior; si bien la participación en los distintos niveles educativos es superior para los hombres que para las mujeres, esta tendencia se revierte en el nivel de educación más alto en el cual las mujeres tienen una participación del 37\% mientras que los hombres tienen una representación del 31\% (gráfico 1).

Gráfico 1. Participación de hombres y mujeres en los niveles educativos en Colombia 2008-II

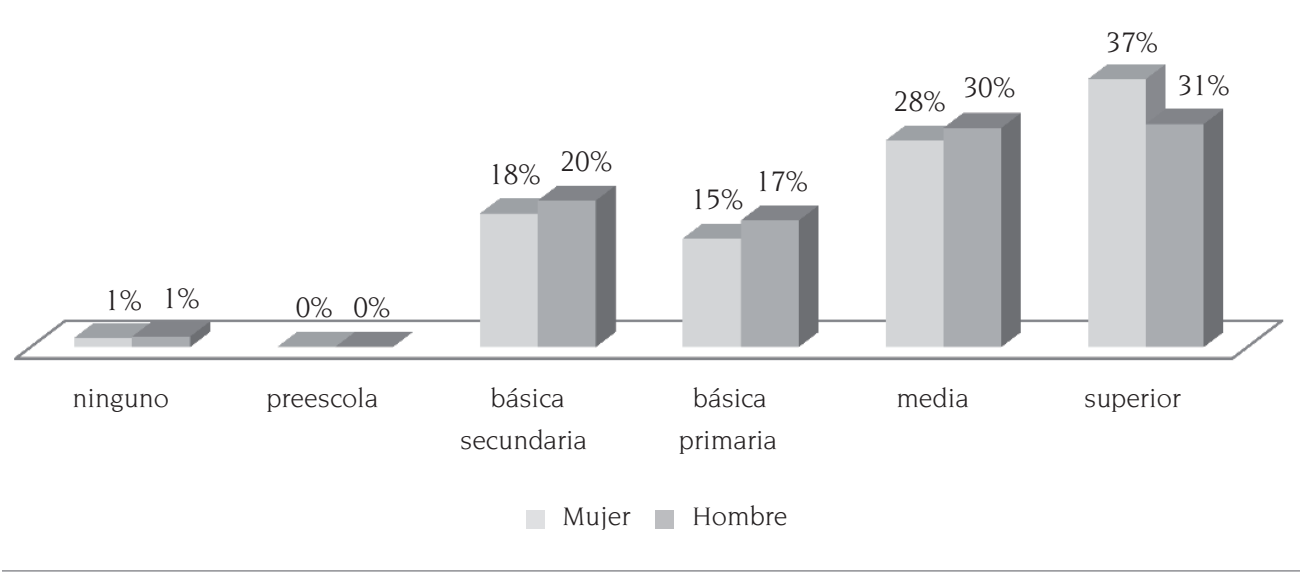

Fuente: elaboración propia con base en la GEIH, 2008-II 
Efectividad y eficiencia de los canales de búsqueda de empleo en Colombia

De acuerdo con la información de la GEIH 2008-II, aquellas personas que se encuentran empleadas han logrado ocuparse en un 73\%, gracias a los canales informales; entre tanto, los desempleados prefieren usar canales formales. Es preciso anunciar que aquí puede estarse en presencia de un sesgo: los canales formales como los avisos clasificados, la entrega de hojas de vida a las empresas y las bolsas de empleo suelen ser medidas utilizadas en última instancia y de acuerdo con la duración del desempleo, estas tenderán a ser empleadas por quienes no tienen empleo, no obstante los canales informales son más efectivos debido a que a los empleados no se les pregunta cuáles canales utilizaron, sino mediante cuál canal obtuvieron el empleo en el que se encuentran laborando.

Esto da paso a otra limitación que vale la pena mencionar: no es lo mismo preguntar por el canal de búsqueda que un sujeto ha empleado para encontrar empleo, que preguntarle cuál le permitió encontrarlo, y si a esto se le suma la necesidad de elegir una sola opción de búsqueda cuando un individuo en realidad utiliza varias, es posible que la distribución de uso de los canales se encuentre recargada a causa de la metodología de recolección de la información; no obstante, como este ejercicio no pretende injerir en las preferencias de los agentes sobre los canales de búsqueda sino verificar la efectividad y la eficiencia de estos, la información, tal como se encuentra disponible, es útil para el ejercicio.

Cuadro 1. Uso de canales de búsqueda por empleados y desempleados en Colombia 2008-II

\begin{tabular}{|l|c|c|c|c|}
\hline \multicolumn{2}{|c|}{ Empleados } & \multicolumn{2}{c|}{ Desempleados } \\
\hline \multicolumn{1}{|c|}{ Canal de búsqueda } & Formales & Informales & Formales & Informales \\
\hline Número de personas & $5.752,62$ & $15.837,38$ & $3.483,62$ & $2.443,38$ \\
\hline Distribución (\%) & $26,64 \%$ & $73,36 \%$ & $58,78 \%$ & $41,22 \%$ \\
\hline
\end{tabular}

Fuente: Cálculos propios con base en la GEIH, 2008-II

Cuadro 2. Uso de los canales de búsqueda desagregados para Colombia 2008-II

\begin{tabular}{|c|l|c|c|l|c|c|}
\hline \multirow{2}{*}{ Tipo de Canal } & \multicolumn{3}{|c|}{ EMPLEADOS } & \multicolumn{3}{c|}{ DESEMPLEADOS } \\
\cline { 2 - 7 } & $\begin{array}{c}\text { ¿Cómo consiguió su } \\
\text { empleo actual? }\end{array}$ & Freq. & $\%$ & $\begin{array}{l}\text { ¿Qué fizo en las ÚLTIMAS } \\
\text { CUATRO SEMANAS para } \\
\text { conseguir empleo? }\end{array}$ & Freq. & $\%$ \\
\hline \multirow{2}{*}{ INFORMALES } & $\begin{array}{l}\text { Pidió ayuda de } \\
\text { familiar, amigos }\end{array}$ & $15.922,54$ & $71 \%$ & $\begin{array}{l}\text { Pidió ayuda de familiar, } \\
\text { amigos }\end{array}$ & $2.325,95$ & $39 \%$ \\
\cline { 2 - 7 } & $\begin{array}{l}\text { Preparativos para } \\
\text { iniciar un negocio }\end{array}$ & NA & NA & $\begin{array}{l}\text { Preparativos para iniciar } \\
\text { un negocio }\end{array}$ & 112 & $2 \%$ \\
\hline
\end{tabular}


Lina María Restrepo Plaza

\begin{tabular}{|c|c|c|c|c|c|c|}
\hline \multirow[b]{2}{*}{ Tipo de Canal } & \multicolumn{3}{|c|}{ EMPLEADOS } & \multicolumn{3}{|c|}{ DESEMPLEADOS } \\
\hline & $\begin{array}{c}\text { ¿Cómo consiguió su } \\
\text { empleo actual? }\end{array}$ & Freq. & $\%$ & $\begin{array}{l}\text { ¿Qué hizo en las ÚLTIMAS } \\
\text { CUATRO SEMANAS para } \\
\text { conseguir empleo? }\end{array}$ & Freq. & $\%$ \\
\hline \multirow{6}{*}{ FORMALES } & $\begin{array}{l}\text { Envió HV a em- } \\
\text { presas }\end{array}$ & $3.094,94$ & $14 \%$ & Envió HV a empresas & $3.031,75$ & $51 \%$ \\
\hline & $\begin{array}{l}\text { Envió HV a bolsas } \\
\text { de empleo o } \\
\text { intermediario }\end{array}$ & 794 & $4 \%$ & $\begin{array}{l}\text { Envió HV a bolsas de } \\
\text { empleo o intermedi }\end{array}$ & 344 & $6 \%$ \\
\hline & Aviso clasificado & 348 & $2 \%$ & Aviso clasificado & 75 & $1 \%$ \\
\hline & $\begin{array}{l}\text { Presentó convoca - } \\
\text { torias }\end{array}$ & $1.096,62$ & $5 \%$ & Presentó convocatorias & 25 & $0 \%$ \\
\hline & $\begin{array}{l}\text { Por el sistema de } \\
\text { información SENA }\end{array}$ & 216 & $1 \%$ & $\begin{array}{l}\text { Por el sistema de infor- } \\
\text { mación SENA }\end{array}$ & NA & NA \\
\hline & Internet & 477,27 & $2 \%$ & Internet & NA & NA \\
\hline OTRO & Otro medio & 410 & $1 \%$ & Otro medio & 68 & $1 \%$ \\
\hline \multicolumn{2}{|l|}{ Total } & $22.359,92$ & $100 \%$ & Total & $5.982,47$ & 100 \\
\hline
\end{tabular}

Fuente: Cálculos propios con base en la GEIH, 2008-II

En el gráfico 2 se puede observar cómo los canales de búsqueda formales incrementan el porcentaje de generación de empleo a medida que aumenta el nivel educativo, y tiene sentido ya que el acceso a convocatorias y a búsquedas en Internet, por ejemplo, son medios de difícil acceso para personas con bajos niveles de educación; así que aunque más adelante se utilizaron los años de educación para realizar las estimaciones de efectividad y eficiencia de los canales, debe reconocerse que puede existir cierta endogeneidad entre el uso de algunas alternativas de búsqueda y el nivel educativo.

Se observa, además, que el uso de canales informales por parte de los desempleados aumenta hasta los niveles de Básica Primaria pero empiezan a reducirse a partir de ahí; esto se debe al tipo de empleo al que se aspira, a las preferencias de los individuos y al acceso que tienen los más educados a canales de búsqueda más formales.

Es interesante el comportamiento en forma de "u" para el uso de canales informales según estrato, por parte de los empleados, presentado en el gráfico 3. En la misma línea de Uribe, Viáfara y Oviedo (2007, p. 51), estratos bajos y estratos muy altos son intensivos en el uso de canales informales, y ambos por razones distintas. Por una parte, las personas de estratos más bajos son menos educadas, lo cual les impide acceder de forma efectiva a las convocatorias y a las búsquedas virtuales, y por otra parte, suelen emplearse en empleos informales y de baja calidad, los cuales son obtenidos a través de canales informales. En cambio, las personas de estrato 
Efectividad y eficiencia de los canales de búsqueda de empleo en Colombia

alto, si bien suelen presentar mayores niveles de capital humano, también gozan de un capital relacional de alta calidad que les permite acceder a empleos que se encuentran acordes con las capacidades técnicas; de ahí que los empleados del estrato 6 utilicen canales informales en un 77\%.

Gráfico 2. Uso de canales de búsqueda según nivel educativo en Colombia 2008-II

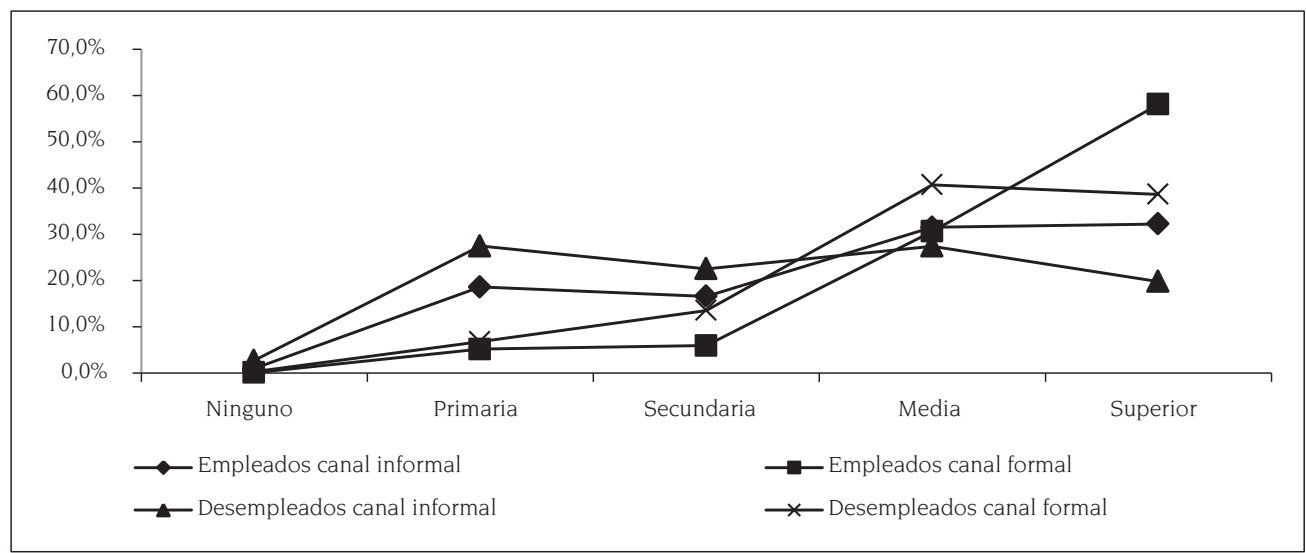

Fuente: Cálculos propios con base en la GEIH, 2008-II

En contraste, el uso de canales formales presenta la forma de una " $u$ " invertida en la cual los empleados de los estratos 4 y 5 , presentan una utilización del 28\% y 26\%, y los desempleados de un 13\% y 10\%, respectivamente. A grandes rasgos se puede decir que los canales formales son los más utilizados por los individuos de estratos 4 y 5 , pero solo la décima parte de ellos obtiene empleo a través de estos canales.

Gráfico 3. Utilización de los canales de búsqueda según estrato en Colombia 2008-II

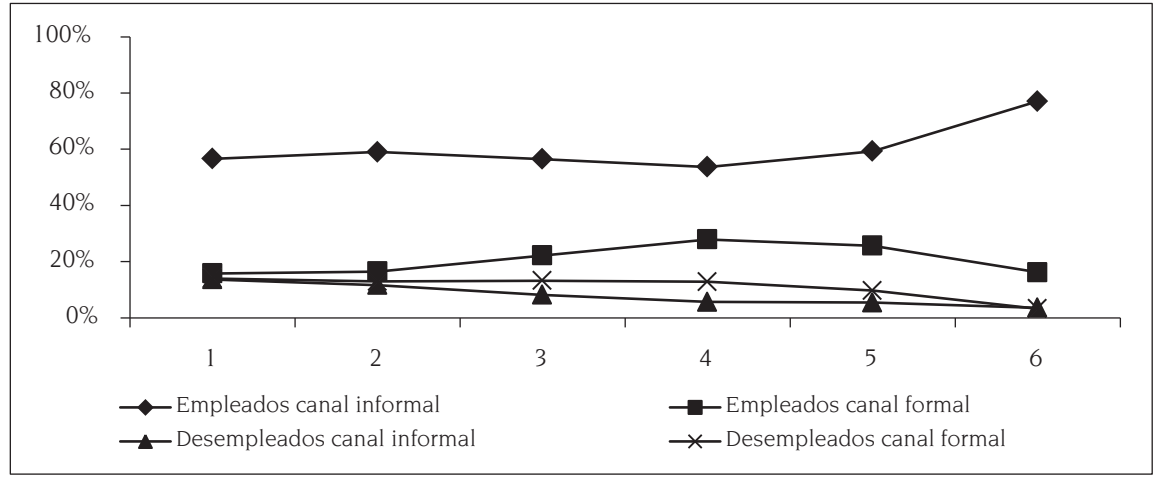

Fuente: Cálculos propios con base en la GEIH, 2008-II

Semestre Económico, volumen 16, №. 33, pp. 67-98 • ISSN 0120-6346, enero-junio de 2013, Medellín, Colombia 
Lina María Restrepo Plaza

En cuanto a la duración media del desempleo de los ocupados (gráfico 4), se observa una duración media de 50,4 semanas, cuando utilizan canales informales, y de 43,8 semanas, cuando utilizan canales formales. Obsérvese que los canales de búsqueda informales generan una mayor duración del desempleo para los empleados que para los desempleados, quienes por definición, en el momento de la encuesta, aún no conocen la duración total del mismo; esto podría ocasionar una subestimación de la duración media del desempleo.

¿Qué puede explicar el hecho de que el 73\% de los empleados encuentre empleo por canales informales aunque tarden más tiempo en emplearse? Los canales informales ofrecen una búsqueda lenta pero segura. Un análisis ingenuo al buscador diría que este no es racional porque el costo de oportunidad de la búsqueda es demasiado alto: ¿por qué insiste? En un país con debilidades institucionales como Colombia, las decisiones individuales son superadas por las condiciones del contexto económico y social.

Es de esperarse, que los canales formales reporten una mayor duración del desempleo para quienes no están empleados; esto se debe a que, como ya se observó antes, el nivel de utilización de estos canales es casi el 60\% y si es persistente la incapacidad de ubicar un empleo para estos sujetos, esto debe incidir en su eficiencia?.

Gráfico 4. Duración media del desempleo según canales para Colombia 2008-II

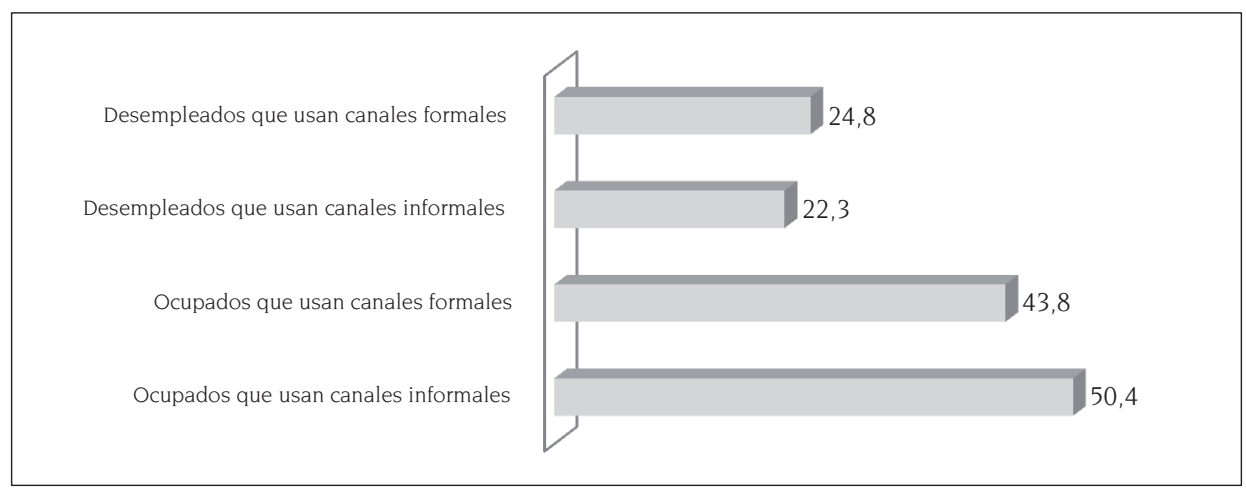

Fuente: Cálculos propios con base en la GEIH, 2008-II

9 Cabe anotar que a diferencia de los empleados, la media de la duración del desempleo no es estadísticamente significativa al $10 \%$ para los desempleados; esto puede deberse a la imposibilidad de capturar la duración exacta del desempleo en el momento de realizar la encuesta (véase anexo 1). 
Efectividad y eficiencia de los canales de búsqueda de empleo en Colombia

\section{LA EFECTIVIDAD DE LOS CANALES DE BÚSQUEDA DE EMPLEO}

Al principio de este documento se mencionó que la efectividad de los canales de búsqueda está asociada a la capacidad que estos tienen para sacar a un individuo del desempleo, bajo la hipótesis de que el entorno socioeconómico influye sobre este fenómeno, razón por la cual el estrato es utilizado como una variable de control.

Un indicador simple de la efectividad corrobora que el estrato socioeconómico influye sobre la posibilidad de encontrar empleo. Sea $e_{j}^{i}$ la proporción de empleados del estrato $j$ que encontraron su empleo mediante canales informales (i) y $d_{j}^{i}$ la proporción de desempleados del estrato $j$ que buscaron empleo mediante estos canales; puede darse cuenta de la efectividad de los canales informales para la consecución de un empleo.

$$
\frac{e_{j}^{i}}{e_{j}^{i}+d_{j}^{i}}
$$

En general, los resultados sugieren que los canales de búsqueda de empleo son más efectivos a medida que aumenta el estrato socioeconómico, y en particular, los canales informales lo son aún más, debido al efecto de las redes sociales de alta calidad.

Se observa, además, que la efectividad de los canales de búsqueda formales tiende a acercarse a la de los canales informales a medida que el estrato se incrementa; la posibilidad de encontrar empleo mediante un canal informal tan solo difiere en quince puntos porcentuales entre una persona de estrato uno y una de estrato 6 , mientras que esta misma diferencia es del doble (treinta puntos) para el caso de los canales formales. ¿Cuáles son las barreras que ocasionan esta desigualdad en las posibilidades de acceso al empleo mediante la búsqueda formal?

Surge pues una reflexión obligada acerca de la importancia de la educación en los grupos más vulnerables de la sociedad para adquirir competencias técnicas que les faciliten la utilización de medios de búsqueda de empleo más meritocráticos. Por otra parte, es necesario promover entre los empresarios la contratación de personal a través de medios formales para lo cual quizá sea necesario instaurar algunas alternativas que reduzcan los costos de transacción asociados a estas actividades.

Los estratos más bajos se encuentran atrapados en una trampa de la pobreza que es difícil de romper, y la evidencia aquí presentada refleja que los procesos de selección en el mercado laboral colombiano inician desde la elección del canal de búsqueda. 
Lina María Restrepo Plaza

Gráfico 5. Efectividad de los canales de búsqueda según estrato en Colombia 2008-II

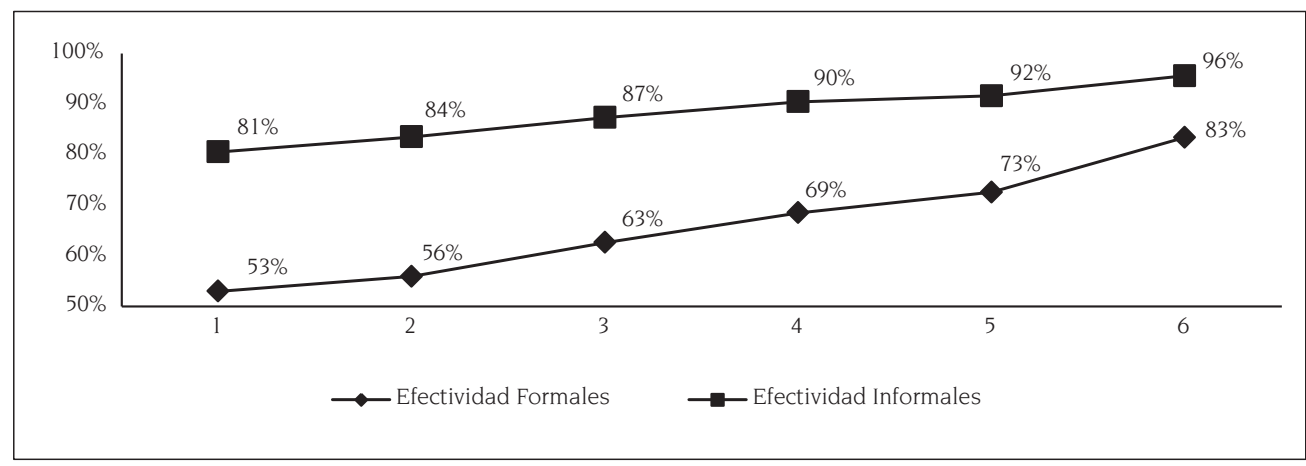

Fuente: cálculos propios con base en la GEIH, 2008-II

Un método más sofisticado para identificar la efectividad de los canales de búsqueda es estimar la probabilidad de conseguir empleo a partir de métodos formales e informales (cuadro 3); para ello se estimó un modelo de elección discreta para cada nivel socio-económico (alto, medio y bajo), el modelo no solo presenta el efecto de los canales de búsqueda en la probabilidad de estar ocupado, sino que, además, intenta verificar el efecto de las variables demográficas del individuo y aquellas variables que determinan su salario de reserva (véanse pruebas de bondad de ajuste en el anexo B).

Se observa que, con relación a los canales informales (categoría de referencia), los canales formales reducen la probabilidad de que un individuo consiga empleo; este efecto es menor a medida que se incrementa el estrato debido a que la calidad de las redes sociales garantiza mejores resultados en los estratos más altos.

Aunque contra-intuitivo, ser hombre reduce la probabilidad de encontrar empleo, sobre todo en el estrato más alto, debido, probablemente, a que la participación de las mujeres es más representativa en los niveles de formación más avanzados, lo cual incrementa el costo de oportunidad de estar desempleadas. Se encontró, además, que la edad y los años de educación incrementan la probabilidad de emplearse. La magnitud de efecto es menor para los estratos bajo y alto; esto puede estar asociado a que los estratos bajos están poco calificados, y los altos suelen reportar un salario de reserva mayor.

La jefatura de hogar afecta en forma directa la probabilidad de estar ocupado; esto se debe a las obligaciones familiares que reposan sobre los jefes de hogar. A medida que aumenta el estrato, las obligaciones se incrementan y, con ellas, el costo de oportunidad de estar desempleado; por eso en estratos más altos 
Efectividad y eficiencia de los canales de búsqueda de empleo en Colombia

es mayor la magnitud del efecto de la jefatura del hogar sobre la probabilidad de estar ocupado.

Por último, la edad en su transformación cuadrática guarda una relación negativa con la posibilidad de ocuparse; esto puede ser ocasionado por los rendimientos decrecientes de acercarse a edades más maduras y por la obsolescencia del capital humano. Esta variable no es significativa en el caso de los individuos de estrato bajo debido a que los sujetos de este nivel socioeconómico pueden salir del ciclo laboral con dificultad, aún si se encontrasen en edades poco aptas para tales fines.

Cuadro 3. Estimaciones para la efectividad de los canales de búsqueda en tres niveles socioeconómicos para Colombia

2008-II10

\begin{tabular}{|l|c|c|c|c|}
\hline \multicolumn{1}{|c|}{ Variable } & General & Estrato Bajo & Estrato Medio & Estrato Alto \\
\hline Ctotal & $-0,17^{* * *}$ & $-0,22^{* * *}$ & $-0,18^{* * *}$ & $-0,17^{* * *}$ \\
\hline Bsexo & $-0,02^{* * *}$ & $-0,01^{*}$ & $-0,025^{* *}$ & $-0,030^{* * *}$ \\
\hline Edad & $0,004^{* * *}$ & $0,006^{* * *}$ & $0,005^{* * *}$ & $0,004^{* * *}$ \\
\hline Bjefe & $0,035^{* * *}$ & $0,03^{* * *}$ & $0,04^{* * *}$ & $0,04^{* * *}$ \\
\hline Añosedu & $0,004^{* * *}$ & $-0,00006^{* * *}$ & $0,004^{* * *}$ & $0,004^{* * *}$ \\
\hline edad2 & $-0,00003^{* * *}$ & $-0,00003$ & $-0,00004^{* * *}$ & $-0,00003^{* * *}$ \\
\hline Ll & $-10.281,35$ & $-953,13$ & $-2914,72$ & $-4005,40$ \\
\hline Bic & $20.637,52$ & $1.964,08$ & $5.894,99$ & $8.079,80$ \\
\hline Aic & $20.576,72$ & $1.920,25$ & $5.843,44$ & $8.024,80$ \\
\hline N & 43.718 & 3.870 & 11.664 & 19.089 \\
\hline
\end{tabular}

Legend: ${ }^{*} \mathrm{p}<.1 ;{ }^{* *} \mathrm{p}<.05 ;{ }^{* * *} \mathrm{p}<.01$

Ctotal, representa el canal de búsqueda de empleo, tomando el valor de uno los canales formales y cero los informales. Bsexo es una variable que describe el sexo del individuo; esta toma el valor de uno si es hombre y cero si es mujer. Edad es una variable continua que representa la edad de cada individuo el día que se le realizó la encuesta. Bjefe es una variable que captura si el individuo es jefe de hogar, toma el valor de uno cuando cumple con esta característica. Añosedu es una variable continua que representa el número de años de educación que tiene cada sujeto. Edad2 es la versión cuadrática de la edad y busca capturar los rendimientos decrecientes de la experiencia. Ll es el criterio de información de la razón de verosimilitud, Bic es el criterio de información bayesiano, Aic, es el Akaike y $\mathbf{N}$ el número de individuos presentes en la estimación.

Fuente: cálculos propios con base en la GEIH, 2008-II

10 La capacidad de predicción de los modelos se encuentra alrededor del 92\%, es decir, el modelo acierta con el $92 \%$ de los casos que se encuentran en la base de datos utilizada. Las medidas de bondad de ajuste se encuentran en el anexo 2.

Semestre Económico, volumen 16, №. 33, pp. 67-98 • ISSN 0120-6346, enero-junio de 2013, Medellín, Colombia 
Lina María Restrepo Plaza

\section{LA EFICIENCIA DE LOS CANALES DE BÚSOUEDA: MODELOS DE DURACIÓN}

Los modelos de duración permitirán dar cuenta de la eficiencia de los distintos canales de búsqueda de empleo. En principio, se realiza un análisis de supervivencia a partir de funciones Kaplan-Meier para determinar la relación existente entre la duración del desempleo y la probabilidad de emplearse. Posteriormente, se estiman 3 modelos de duración para reconocer la distribución más adecuada según el proceso generador de los datos para los dos canales de búsqueda considerados.

\subsection{Análisis de supervivencia Kaplan-Meier: no paramétrico}

En el caso de los modelos no paramétricos se realizó una estimación Kaplan-Meier que arrojó resultados intuitivos acerca de la probabilidad de sobrevivir en una situación de desempleo. La intuición estadística para este tipo de estimaciones es sencilla: para estar desempleados durante un año, es necesario estar desempleado durante 52 semanas; calculando los sucesos de desempleo que permanecen en cada semana, se obtiene la probabilidad de sobrevivir en dicha situación, segmentando el análisis de acuerdo con los dos canales de búsqueda: formales e informales.

A través de un análisis no paramétrico tipo Kaplan-Meier, se puede contrastar, de forma exploratoria, la probabilidad de permanecer en una situación de desempleo a medida que pasa el tiempo. La permanencia en el desempleo tiende a presentar una relación negativa con respecto al tiempo, y esta relación puede ser distinta de acuerdo con los canales de búsqueda empleados.

Gráfico 6. Duración del desempleo según canales

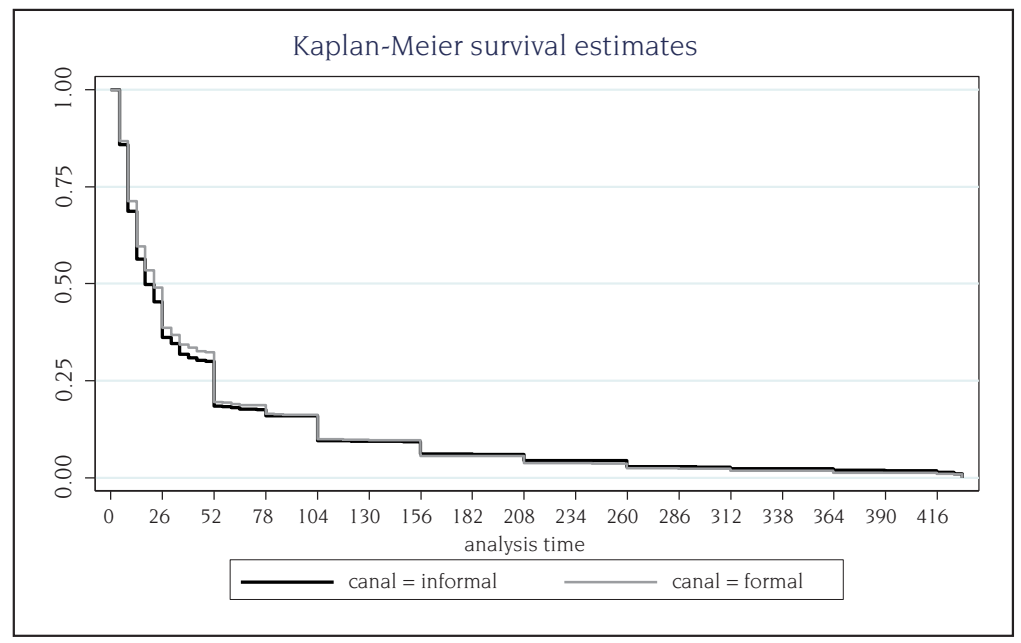

Fuente: Cálculos propios con base en la GEIH, 2008-II 
Efectividad y eficiencia de los canales de búsqueda de empleo en Colombia

Se observa que, en términos generales, la duración del período de desempleo presenta un decrecimiento monótono por lo que la probabilidad de permanecer desempleado se reduce a medida que pasa el tiempo. Aunque en magnitudes marginales, los canales de búsqueda formales parecen retrasar más la posibilidad de obtener un empleo. En particular, mientras que al utilizar canales informales, un cesante tiene una probabilidad de encontrar empleo a las 13 semanas del 43,63\%, un individuo que utiliza canales formales encuentra empleo con una probabilidad del $40,44 \%$.

\subsection{Análisis paramétrico de la duración del desempleo}

Para el caso de los modelos paramétricos, se estimó un modelo de duración a través del método de máxima verosimilitud para una distribución que se ajuste al desempeño de los datos. Por supuesto, la variable dependiente es el tiempo (en semanas) que un sujeto ha estado o estuvo buscando empleo.

De acuerdo con el comportamiento observado de la función de supervivencia, se puede asumir que la distribución de la misma debe ser monótona y decreciente, tal como lo son las distribuciones Weibull y la Gompertz. Después de realizar unas pruebas con la función Gamma se evidenció que los parámetros no coincidían con los de una distribución Weibull, razón por la cual se utilizó la distribución Gompertz para estimar el modelo de supervivencia ${ }^{11}$.

De acuerdo con la estimación se puede decir que los canales formales reducen la probabilidad de salir del desempleo en el tiempo. Ser hombre y ser jefe de hogar hace que sea probable conseguir empleo más pronto, y esta probabilidad aumenta si se utilizan canales informales; esto se debe a que las responsabilidades del hogar reducen el salario de reserva del individuo y, por lo tanto, lo hacen propenso a aceptar más rápido una oferta de empleo.

Sujetos mayores salen del desempleo más rápido; sin embargo, esta variable no es significativa para los canales informales, pues, al parecer, el efecto de las redes sociales es similar para sujetos de distintas edades; esto quiere decir que si un sujeto de 20 años y otro de 40 años le solicitan a un mismo amigo con dos vacantes disponibles, el vínculo fuerte les permitirá a ambos obtener el empleo. Puede observarse que la expresión cuadrática de la edad disminuye la probabilidad de encontrar empleo con el tiempo; los sujetos pierden habilidades que no se compensan con el conocimiento adquirido a través de los años.

11 Las pruebas se encuentran en el anexo C 
Lina María Restrepo Plaza

Los años de educación parecen reducir la probabilidad de emplearse con el tiempo para individuos que utilizan los canales informales; eso se encuentra asociado al incremento en el salario de reserva de las personas mejor cualificadas, las cuales se encuentran en estratos más altos en donde los canales informales suelen ser más efectivos debido a la calidad de las redes sociales. La variable educación no resultó significativa para los canales de búsqueda formales lo que puede estar asociado a la endogeneidad antes mencionada entre la utilización de este tipo de canales y unos requerimientos mínimos de conocimientos técnicos para la búsqueda a través de ellos.

Cuadro 4. Estimadores y criterios de Akaike para el modelo de duración

\begin{tabular}{|l|c|c|c|}
\hline \multicolumn{1}{|c|}{ Variable } & Modelo General & Canales Formales & Canales informales \\
\hline Canal & $0,93^{* * *}$ & - & - \\
\hline Bsexo & $1,43^{* * *}$ & $1,21^{* * *}$ & $1,53^{* * *}$ \\
\hline Edad & $1,02^{* * *}$ & $1,08^{* * *}$ & 1,0005 \\
\hline Bjefe & $1,24^{* * *}$ & $1,23^{* * *}$ & $1,24^{* * *}$ \\
\hline Añosedu & $0,99^{* * *}$ & 1,0004 & $0,99^{* * *}$ \\
\hline Edad2 & $0,99^{* * *}$ & $0,999^{* * *}$ & $0,99^{* * *}$ \\
\hline Estrato Medio & 0,98 & 1,01 & 0,97 \\
\hline Estrato Alto & $1,17^{* * *}$ & 1,06 & $1,22^{* * *}$ \\
\hline Akaike & $5.4314,22$ & $8.274,91$ & $2.0476,24$ \\
\hline Gamma $(\gamma)$ & $-0,005$ & $-0,005$ & $-0,005$ \\
\hline
\end{tabular}

Legend: " $\mathrm{p}<0,1 ;{ }^{* *} \mathrm{p}<0,05 ;{ }^{* * *} \mathrm{p}<0,01$

Canal, representa el canal de búsqueda de empleo, tomando el valor de uno los canales formales y cero los informales. Bsexo es una variable que describe el sexo del individuo; esta toma el valor de uno si es hombre y cero si es mujer. Edad es una variable continua que representa la edad de cada individuo el día que se le realizó la encuesta. Bjefe es una variable que captura si el individuo es jefe de hogar, toma el valor de uno cuando cumple con esta característica. Añosedu es una variable continua que representa el número de años de educación que tiene cada sujeto. Edad2 es la versión cuadrática de la edad y busca capturar los rendimientos decrecientes de la experiencia. Estrato medio es una variable discreta que registrar a aquellos individuos que pertenecen al estrato medio, así como Estrato Alto captura a quienes pertenecen a esta categoría. Aic, es el Akaike y Gamma es el criterio de información que describe la tendencia de la función, creciente o decreciente.

Fuente: Cálculos propios con base en la GEIG 2008-II, procesamiento en STATA

Por otra parte, pertenecer al estrato medio no tiene ningún efecto significativo en el tiempo de búsqueda de empleo, mientras que formar parte de un estrato social alto resulta significativo en el modelo general y en los canales informales; esto se encuentra asociado a que los canales informales son utilizados, en su mayoría, por individuos de estratos altos que poseen redes efectivas para minimizar la permanencia en el desempleo. 
Efectividad y eficiencia de los canales de búsqueda de empleo en Colombia

\section{LAS TAREAS PENDIENTES DEL MERCADO LABORAL COLOMBIANO}

Los hallazgos obtenidos, aunque en su mayoría esperados, son una oportunidad para llamar la atención sobre dos resultados bastante sugestivos:

- Los canales formales no son rivales pero sí excluyentes: ¿̇on un monopolio natural?

- Los canales formales reducen el tiempo de búsqueda para los empleados; y aunque no ocurre lo mismo para los desempleados, tampoco se puede decir a ciencia cierta que los desempleados salgan más rápido del paro a través de canales informales: los desempleados no saben cuándo ni cómo obtendrán empleo.

\subsection{Desde la oferta de canales de búsqueda de empleo}

Los monopolios naturales son aquellas estructuras de mercado en las cuales el costo de producir una unidad adicional es decreciente, por esta razón el hecho de que otra persona se presente a una convocatoria no incrementa los costos de selección del empresario; sin embargo, existen barreras desde la oferta y desde la demanda para la utilización de este tipo de canales, y como se hace mención al uso de los canales, no se hace referencia a la oferta y demanda de empleo, sino a la de los mecanismos de búsqueda y selección de trabajadores, y en ese sentido se debe establecer una restricción: un buscador solo utilizará un canal informal, por ejemplo, si un empresario establece que el proceso de selección se realizará a través de medios informales. Lo anterior es también válido para los canales formales; en otras palabras, el evento de búsqueda de empleo solo se activa si existe un matching entre los métodos de buscadores y empresarios.

No todos los empleos son susceptibles de someterse a procesos de búsqueda formal. Por ejemplo, el Ministro del Interior del país no tuvo que llevar su hoja de vida a la Oficina de Recursos Humanos del Palacio de Nariño para someterse a una convocatoria para que lo consideraran como candidato en el proceso de selección para el Ministerio; no tuvo que aplicar a una convocatoria ni mucho menos ojear los avisos clasificados. ¿Por qué? Porque es un personaje de la vida pública, con reconocimiento dentro de las esferas políticas y, gracias a su conocido desempeño, nadie debería objetar su nombramiento. Si se hubiera realizado una convocatoria existiría una altísima probabilidad de incurrir en problemas de riesgo moral y selección adversa; por eso es mejor hacer uso de las redes establecidas y de la confianza que provee conocimiento previo. El costo social de una mala gestión del Ministro es muy alto; de forma similar sucede con los presidentes de las grandes empresas del mundo. 
Lina María Restrepo Plaza

Tampoco se realiza una convocatoria para elegir a la persona que le ayudará con las ventas de su tienda familiar, y existen varias razones para ello. En primer lugar, los negocios pequeños son, por lo general, unidades financieramente frágiles, las actividades a desarrollar son sencillas y el personal necesario es poco. Se prefiere, pues, contratar personas conocidas para eliminar el riesgo de selección adversa y para poder llegar acuerdos implícitos de una contratación informal, es decir, por fuera de los parámetros de la ley. Además, un proceso de búsqueda mediante canales formales requiere de costos de entrada que un pequeño empresario no estaría dispuesto a asumir: un aviso clasificado, un aplicativo para la suscripción de hojas de vida web, un experto en procesos de selección, entre otros.

Los últimos párrafos describen dos situaciones extremas, en las cuales la utilización de canales de búsqueda informales es un método socialmente válido cuando los costos de transacción y las erogaciones directas para poner a funcionar un proceso formal son muy altos. No obstante, es difícil creer que los empleos de la economía colombiana fluctúen entre estos dos escenarios, pero lo que sí es un hecho es que el $60 \%$ de la población de este país se encuentra en la informalidad, y que en esa cifra no están los ministros ni los presidentes de las multinacionales, sino el colombiano que acude a amigos, vecinos o familiares para ocuparse, o hace uso de ahorros o créditos para llevar a cabo algún emprendimiento productivo.

La economía colombiana requiere de una política con una visión más sistémica de lo que el país necesita. El país no necesita exenciones tributarias para incentivar la creación de puestos de trabajo porque la demanda laboral no depende de los impuestos: si un empresario necesita producir, va a demandar empleo. Tampoco se requiere flexibilidad total para la imposición del salario porque el empresario no va a contratar más personas de las que necesita solo porque puede pagarles poco; sin embargo, una flexibilización total sí generaría un detrimento total en los ingresos del grueso de los colombianos y por consiguiente en su calidad de vida. Pero entonces, ¿qué necesita el país?

En el país se necesita incrementar las posibilidades de acceso a los beneficios sociales que ofrece el mercado laboral formal; esto es, velar por la efectividad y la eficiencia de la prestación de servicios de salud en el régimen subsidiado y crear un fondo de compensación para las familias que no tienen una contratación laboral con los requisitos de ley. Adicionalmente, se requiere estimular la utilización de vías formales para la contratación de los mandos altos, medios y bajos en las empresas medianas y grandes. Para ello se propone, por una parte, reducir los costos asociados a los procesos de selección para las empresas medianas mediante el fortalecimiento 
Efectividad y eficiencia de los canales de búsqueda de empleo en Colombia

y difusión de las bolsas de empleo públicas; y por otra, reglamentar la contratación meritocrática en los mandos que así lo amerite en las empresas medianas y grandes; esto no solo favorecerá la institucionalidad del país, sino que deberá redundar también en incrementos en la productividad de la economía.

\subsection{Desde la demanda de canales de búsqueda de empleo}

Si el país lograra establecer los incentivos necesarios para que los empresarios realicen procesos de selección a través de mecanismos formales ċuién garantiza que estos mecanismos sean accesibles para el grueso de la población?

A lo largo del documento se pudo evidenciar que los canales de búsqueda formales son más efectivos en los estratos sociales más altos; esto se debe a que son individuos más educados y aspiran a empleos que son susceptibles de asignarse por métodos formales de selección. Pero, además, los segmentos más pobres de la población carecen de los conocimientos técnicos y de los medios económicos para beneficiarse de la información pública; de ahí que es necesario emprender campañas para la estandarización en la utilización de herramientas informáticas básicas.

También es indispensable fortalecer la calidad de la Educación Primaria y secundaria de los colegios públicos y desarrollar programas integrales que inserten a los niños y adolecentes en dinámicas deportivas y culturales que los alejen de la violencia y el consumo de sustancias psicoactivas. Esto romperá vacíos estructurales en los hogares más pobres, favorecerá las posibilidades de acceso a la educación universitaria y acercará a la población vulnerable a las posibilidades de empleo mejor remunerado, en el marco de una economía que asigna recursos de forma meritocrática.

\subsection{La solución no es única}

La respuesta al desarrollo económico no es única, es integral, y aquí solo se han establecido algunas propuestas que parten de una perspectiva que retoma las dinámicas laborales en el país desde el proceso de búsqueda de empleo. Sin embargo, se realiza un intento, un poco arriesgado, por relacionar el proceso de búsqueda con la estructura empresarial del país y con las limitaciones de acceso vía contexto social.

La propuesta es que se piense el desempeño económico desde una visión sistémica. No se debe perder de vista que los individuos responden a incentivos y que todas las decisiones tienen asociado un costo de oportunidad, sin caer en la trampa de pensar que los pobres tienen un costo de oportunidad más bajo. 
Lina María Restrepo Plaza

\section{CONCLUSIONES}

De acuerdo con las estimaciones realizadas, los canales informales suelen ser más efectivos y eficientes en el proceso de búsqueda de empleo; no obstante lo anterior, debe considerarse el posible sesgo generado por las dificultades de conocer a ciencia cierta la duración del desempleo de los desempleados y por la imposibilidad de conocer todos los canales de búsqueda utilizados, de forma simultánea, por los empleados.

Si bien ser hombre reduce la probabilidad de encontrar empleo y más aún en los estratos altos debido a la mayor participación de las mujeres en altos niveles de formación, se encontró también que una vez se entra en una situación de desempleo, ser mujer reduce las probabilidades de salir en el tiempo, lo cual puede estar asociado a incrementos en el salario de reserva generados por la situación anterior.

Por otra parte, se encontró que la edad y los años de educación incrementan la probabilidad de emplearse, siendo la magnitud del efecto menor para los estratos bajo y alto; lo anterior puede deberse a barreras físicas y técnicas para el tipo de trabajos asociados a los estratos bajos, y a límites salariales impuestos por sujetos de estratos altos. Para aquellos que utilizan canales formales, la edad permite salir del desempleo de manera más rápida debido, quizá, a que sujetos más maduros han tenido la posibilidad de alcanzar un mayor nivel de experiencia lo cual les garantiza la posibilidad de salir del desempleo mediante estos canales. No sucede lo mismo con los canales informales, caso en el cual la edad no es significativa debido a que esta variable no parece incidir en el desempeño de las redes sociales.

Si bien los años de educación incrementan la probabilidad de emplearse, también parecen reducir la probabilidad de hacerlo a medida que pasa el tiempo, sobre todo para individuos que utilizan los canales informales; eso se encuentra asociado al incremento en el salario de reserva de las personas mejor cualificadas, las cuales se encuentran en estratos más altos en donde los canales informales suelen ser más efectivos debido a la calidad de las redes sociales. La variable educación no resultó significativa para el canal de búsqueda formal debido, quizá, a la endogeneidad antes mencionada entre la utilización de este tipo de canales y unos requerimientos mínimos de conocimientos técnicos.

Puede observarse que la expresión cuadrática de la edad reduce la probabilidad de ocuparse y de encontrar empleo a medida que pasa el tiempo, ya que los sujetos pierden habilidades que no se compensan con el conocimiento adquirido a través de los años.

Por una parte, a medida que se incrementa el estrato socioeconómico, la duración del desempleo tiende a disminuir en términos generales; esto también se cumple en individuos que hacen uso de los canales informales, ya que incrementos en la 
Efectividad y eficiencia de los canales de búsqueda de empleo en Colombia

condición social les facilitan desarrollar redes sociales generadoras de empleos. En el caso de los canales formales, esta variable no resultó significativa debido a que, como ya se dijo antes, acceder a estos canales requiere de unos conocimientos técnicos mínimos que no son propios de los estratos más bajos. Por otra parte, si bien los sujetos de estratos altos podrían acceder a ellos, se ha visto que presentan preferencias por los canales informales debido a su capital social.

Para finalizar, el país tiene muchas tareas por resolver, pero en este trabajo se destaca la importancia de incentivar, desde la oferta y la demanda, la utilización de canales de búsqueda formales para fortalecer la institucionalidad del país e incrementar los niveles de productividad del mismo. Para ello, desde la oferta se requiere generar incentivos precisos que reduzcan los costos de transacción y las erogaciones directas relacionadas con la instauración de mecanismos de selección formales. Desde la demanda se sugiere diseñar estrategias pedagógicas para la estandarización en conocimientos informáticos básicos y plantear un proceso educativo integral que capture al individuo desde la primera infancia y le otorgue toda la formación para acceder a empleos que le permitan romper los círculos viciosos de la pobreza.

\section{BIBLIOGRAFÍA}

Castellar, C. y Uribe J. I. (2003). Determinantes de la duración del desempleo en el Área Metropolitana de Cali, 1988-2000. En: Archivos de Economía, No. 218, Departamento Nacional de Planeación, marzo de 2003, p. 42.

Du F.L., Yang. J., y Dong X.Y. (2007), Why Women have Longer Unemployment Durations than Men in Post-Restructuring Urban China? [En línea] PMMA Working Paper 2007-23, Julio de 2007, p. 48. <http://www.iza.org/conference_files/worldb2007/dong _ x3377.pdf>. [Consultado: 4 de junio de 2010]

Grenee, W. (2003). Econometric Analysis. Quinta Edición, Prentice Hall, New York University, USA, $1024 \mathrm{p}$.

Kaplan, E y Meier, P. (1958). Nonparametric Estimation from Incomplete Observations. En: Journal of the American Statistical Association, Vol. 53, N. ${ }^{\circ}$ 282, pp. 457- 481.

Kiefer, N. (1988). Economic Duration Data and Hazard Functions. En: Journal of Economic Literature, Vol. 26, N. ${ }^{\circ}$, pp. 646-679.

Lindbeck, A. Y Snower, D., (1986), Wage Setting, Unemployment and Insider-Outsider Relations. En: American Economic Review, Vol. 76, N. ${ }^{\circ}$ 2, pp. 235-240.

Lindbeck, A. Y Snower, D. (1988). The Insider-Outsider Theory of Employment and Unemployment, The MIP Press, Cambridge, Massachusetts, pp. 365-369.

Martin, J.L. (1995), Paro y búsqueda de empleo. Una aproximación desde la teoría económica. Universidad de Sevilla, p. 195

Semestre Económico, volumen 16, №. 33, pp. 67-98 • ISSN 0120-6346, enero-junio de 2013, Medellín, Colombia 
Lina María Restrepo Plaza

Mortensen, D. T., y Pissarides. C. (1994). Job Creation and Job Destruction in the Theory of Unemployment. En: Review of Economic Studies, Vol. 61, N. ${ }^{\circ}$ 3, Julio 1994. pp. 397-415.

Mortensen, D.T. y Pissarides. C (1998). New Developments in Models of Search in the Labor Market, En: Handbook of Labor Economics. Vol. 3, N. ${ }^{\circ}$ 3, p. 74

Oviedo, M. (2002). Optimización Dinámica en Tiempo Discreto. Ecuación de Bellman, [En línea] p. 12. < http://www.eco.unc.edu.ar/ief/miembros/archivos/prof _oviedo/oviedo_optimizacindinmicatiemdiscr.pdf $>$. [Consultado: 18 de junio de 2010]

Oviedo, Y., M. (2007). Canales de búsqueda de empleo y duración del desempleo en el mercado laboral Colombiano 2003. En: Sociedad y Economía, No. 13, Facultad de Ciencias Sociales y Económicas, Universidad del Valle, Cali, pp. 153-173.

Pissarides, C. (2009). The Unemployment Volatility Puzzle: is Wage Stickiness the Answer? En: Econometrica, Vol. 77, N. ${ }^{\circ}$ 5, pp. 1339-1369.

Reid. G.L. (1972). Job Search and the Effectiveness of Job-Finding Methods. [En línea] Industrial and Labor Relations Review, Vol. 25, N. ${ }^{\circ}$, julio de 1972, pp. 479-495.

Roschin S.y. Markova K.V. (2004), Choice among Different Job Search Channels: The Evidence from Russian Labor Market [En línea] Economics Education and Research Consortium, No 04/05, p. 46. < http://pdc.ceu.hu/archive/00001933/>. [Consultado: 4 de junio de 2010]

Stigler, G. (1961). The economics of information En: Journal of Political Economy, Vol. 69, N. 3 , pp. $213-225$.

Tasci, M. (2008). Job Search and Determinants of Job Search Intensity in Turkey. [En línea] Departamento de Econometría de la Universidad de la Universidad de Balikesir, p. 29. < http:// papers.ssrn.com/sol3/papers.cfm?abstract_ $i d=1082715>$. [Consultado: 4 de junio de 2010]

Tenjo J. y Ribero R., (1998). Participación, desempleo y mercados laborales en Colombia, DNP. En: Archivos de Macroeconomía, N. 81, pp. 1-78.

Uribe J., I., Viáfara C., A. y Oviedo Y., M., (2007). Efectividad de los canales de búsqueda de empleo en Colombia en el año 2003. En: Lecturas de Economía, N. ${ }^{\circ}$ 67, Facultad de Ciencias Económicas, Universidad de Antioquia, Medellín, pp. 43-70.

Van Den Berg, G. y Van Der Kaauw, B. (2007). Structural Empirical Evaluation of Job Search Monitoring, [En línea] University Amsterdam, 23 p. <http://client.norc.org/jole/SOLEweb/9168. pdf>. [Consultado: 11 de junio de 2010]

Viáfara C. y Uribe, I. (2009). Duración del Desempleo y Canales de Búsqueda de Empleo en Colombia, 2006. En: Revista de Economía Institucional, Vol. 11, N. 21, 2009, pp. 139-160, Universidad Externado de Colombia.

Weiss, A. (1980). Job Queues and Layoffs in Labor Markets with Flexible Wages. En: Journal of Political Economy, Vol. 88, N.3, junio 1980, pp. 526-538. 
ANEXOS

\section{Anexo A: Test de diferencia de medias en los tiempos de duración}

Para desempleados: de acuerdo con la prueba, no existe suficiente evidencia estadística para rechazar la hipótesis de que la media de la duración del desempleo difiera cuando se busca por canales formales y por canales informales, razón por la cual, la duración puede considerarse igual para ambos.

Cuadro 1. Test de diferencia de medias para los desempleados

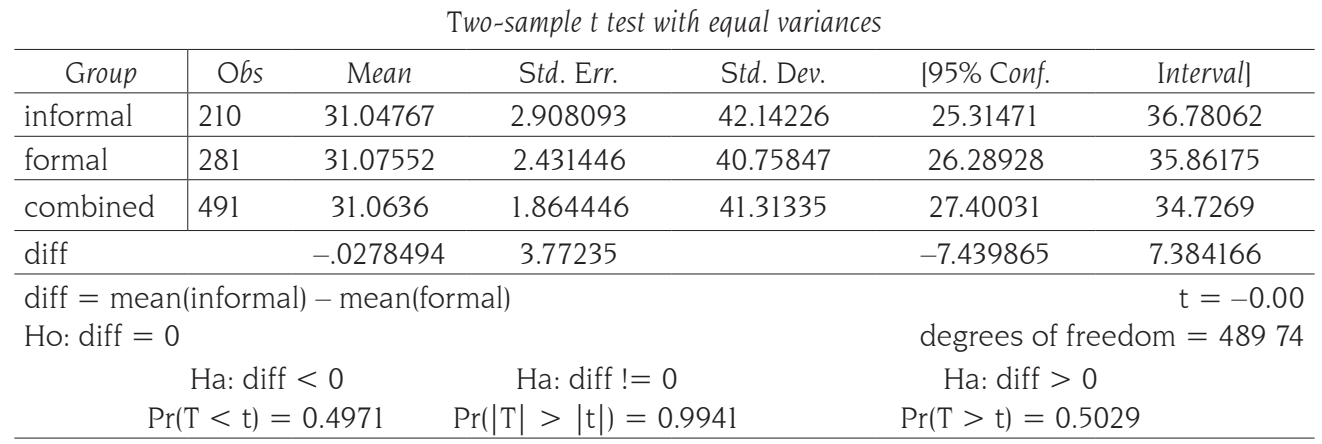

Fuente: cálculos propios con base en la GEIG 2008-II, procesamiento en STATA

Para empleados: de acuerdo con la prueba, existe suficiente evidencia estadística para rechazar la hipótesis de que la media de la duración del desempleo difiere cuando se busca por canales formales y por canales informales, razón por la cual la duración puede considerarse diferente según el canal de búsqueda utilizado.

.ttest tbusc if ocu $==1$, by (canal)

Cuadro 2. Test de diferencia de medias para los empleados

Two-sample t test with equal variances

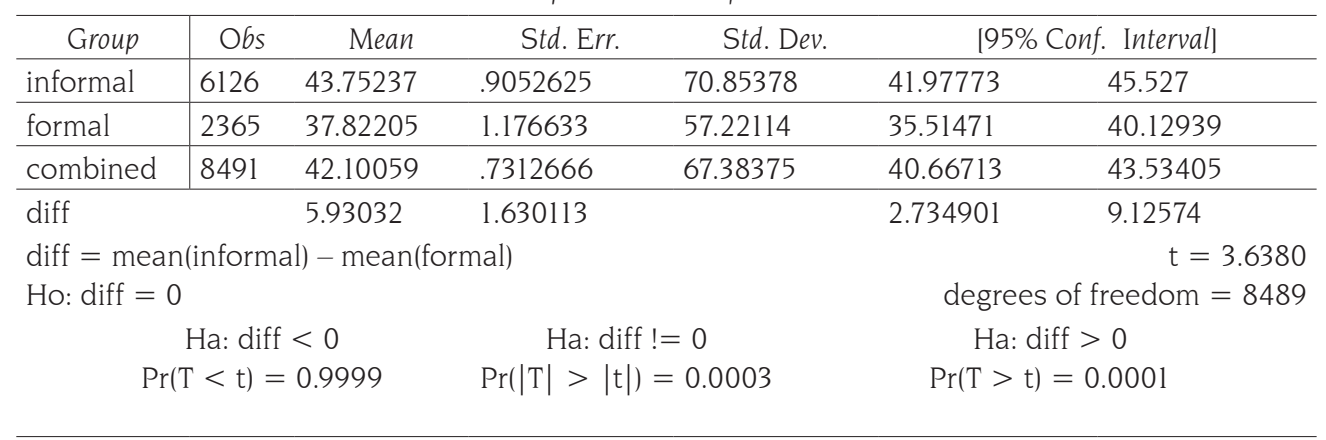

Fuente: cálculos propios con base en la GEIG 2008-II, procesamiento en STATA 
Lina María Restrepo Plaza

\section{Anexo B: Medidas de bondad de ajuste de los modelos de efectividad}

La capacidad de predicción de los modelos se encuentra alrededor del 92\%, es decir, el modelo acierta con el $92 \%$ de los casos que se encuentran en la base de datos utilizada. De acuerdo con los resultados de la prueba Chi-cuadrado de Pearson, se observa que el modelo se ajustado es significativo para la estimación general y la para estimación en estratos bajos. La prueba de Hosmer y Lemeshow arroja que el modelo se encuentra bien especificado para el caso general y para el estrato alto.

Cuadro 3. Medidas de bondad de ajuste para las estimaciones de la efectividad de los canales de búsqueda en tres niveles socioeconómicos

\begin{tabular}{|c|c|c|c|c|}
\hline \multirow{2}{*}{ PRUEBA } & \multicolumn{4}{|c|}{ VALOR } \\
\hline & General & Estrato Bajo & Estrato Medio & Estrato Alto \\
\hline $\begin{array}{l}\text { Cociente de } \\
\text { verosimilitud }\end{array}$ & 0,1228 & 0,1310 & 0,1151 & 0,1237 \\
\hline $\begin{array}{l}\text { Chi-cuadrado } \\
\text { de Pearson }\end{array}$ & $\begin{array}{c}\text { Pearson } \\
\text { chi2 } 2(5885)=6371.72 \\
\text { Prob }>\text { chi2 }=0.0000\end{array}$ & $\begin{array}{c}\text { Pearson } \\
\text { chi2 }(1758)=1856.62 \\
\text { Prob }>\text { chi } 2=0.0501\end{array}$ & $\begin{array}{c}\text { Pearson } \\
\text { chi2 }(3278)=3217.31 \\
\text { Prob }>\text { chi } 2=0.7722\end{array}$ & $\begin{array}{c}\text { Pearson } \\
\text { chi2 } 2(4264)=4513.85 \\
\text { Prob }>\text { chi } 2=0.0039\end{array}$ \\
\hline $\begin{array}{l}\text { Porcentaje de } \\
\text { aciertos del } \\
\text { modelo }\end{array}$ & $92.46 \%$ & $91.85 \%$ & $91.9 \%$ & $92.32 \%$ \\
\hline $\begin{array}{l}\text { Prueba de } \\
\text { Hosmer- } \\
\text { Lemeshow }\end{array}$ & $\begin{array}{c}\text { Hosmer-Lemeshow } \\
\operatorname{chi} 2(8)=33.73 \\
\text { Prob }>\text { chi } 2=0.0000\end{array}$ & $\begin{array}{c}\text { Hosmer-Lemeshow } \\
\text { chi2 } 2(8)=4.78 \\
\text { Prob }>\text { chi } 2=0.7809\end{array}$ & $\begin{array}{c}\text { Hosmer-Lemeshow } \\
\text { chi2 }(8)=5.73 \\
\text { Prob }>\text { chi } 2=0.6769\end{array}$ & $\begin{array}{c}\text { Hosmer-Lemeshow } \\
\operatorname{chi} 2(8)=27.54 \\
\text { Prob }>\text { chi } 2=0.0006\end{array}$ \\
\hline
\end{tabular}

Fuente: cálculos propios con base en la GEIG 2008-II, procesamiento en STATA 
Efectividad y eficiencia de los canales de búsqueda de empleo en Colombia

\section{Anexo C: Pruebas para la elección de la mejor distribución para el modelo de duración}

Cuadro 4. Pruebas Chi2 para el valor de Kappa en los modelos de duración

\begin{tabular}{|c|c|c|c|c|c|c|}
\hline \multicolumn{3}{|c|}{ General } & \multicolumn{2}{|c|}{ Formales } & \multicolumn{2}{|c|}{ Informales } \\
\hline & $\mathrm{K}=0$ & $\mathrm{~K}=1$ & $K=0$ & $\mathrm{~K}=1$ & $K=0$ & $\mathrm{~K}=1$ \\
\hline Chi2(1) & 336.54 & 2571.69 & 99.49 & 546.79 & 269.33 & 1409.54 \\
\hline Prob $>$ chi 2 & 0.0000 & 0.0000 & 0.0000 & 0.0000 & 0.0000 & 0.0000 \\
\hline $\begin{array}{l}\text { Conclusión } \\
\text { sobre la } \\
\text { distribución }\end{array}$ & $\begin{array}{l}\text { Se rechaza la } \\
\text { hipótesis de } \\
\text { que K=0, por } \\
\text { lo que no exis- } \\
\text { te suficiente } \\
\text { evidencia para } \\
\text { afirmar que el } \\
\text { mejor modelo } \\
\text { paramétrico } \\
\text { cumpla una } \\
\text { distribución } \\
\text { tipolognormal. }\end{array}$ & $\begin{array}{l}\text { Se rechaza la } \\
\text { hipótesis de } \\
\text { que K=1, por } \\
\text { lo que no exis- } \\
\text { te suficiente } \\
\text { evidencia para } \\
\text { afirmar que el } \\
\text { mejor modelo } \\
\text { paramétrico } \\
\text { cumpla una } \\
\text { distribución } \\
\text { tipo Weibull. }\end{array}$ & $\begin{array}{l}\text { Se rechaza la } \\
\text { hipótesis de } \\
\text { que K=0, por } \\
\text { lo que no exis- } \\
\text { te suficiente } \\
\text { evidencia para } \\
\text { afirmar que el } \\
\text { mejor modelo } \\
\text { paramétrico } \\
\text { cumpla una } \\
\text { distribución } \\
\text { tipolognormal. }\end{array}$ & $\begin{array}{l}\text { Se rechaza la } \\
\text { hipótesis de } \\
\text { que } \mathrm{K}=1 \text {, por } \\
\text { lo que no exis- } \\
\text { te suficiente } \\
\text { evidencia para } \\
\text { afirmar que el } \\
\text { mejor modelo } \\
\text { paramétrico } \\
\text { cumpla una } \\
\text { distribución } \\
\text { tipo Weibull. }\end{array}$ & $\begin{array}{l}\text { Se rechaza la } \\
\text { hipótesis de } \\
\text { que } K=0 \text {, por } \\
\text { lo que no exis- } \\
\text { te suficiente } \\
\text { evidencia para } \\
\text { afirmar que el } \\
\text { mejor modelo } \\
\text { paramétrico } \\
\text { cumpla una } \\
\text { distribución } \\
\text { tipolognormal. }\end{array}$ & $\begin{array}{l}\text { Se rechaza la } \\
\text { hipótesis de } \\
\text { que } \mathrm{K}=1 \text {, por } \\
\text { lo que no exis- } \\
\text { te suficiente } \\
\text { evidencia para } \\
\text { afirmar que el } \\
\text { mejor modelo } \\
\text { paramétrico } \\
\text { cumpla una } \\
\text { distribución } \\
\text { tipo Weibull. }\end{array}$ \\
\hline
\end{tabular}

Fuente: cálculos propios con base en la GEIG 2008-II, procesamiento en STATA 